\title{
Charging of Multiple Interacting Particles by Contact Electrification
}

\section{Citation}

Soh, Siowling, Helena Liu, Rebecca Cademartiri, Hyo Jae Yoon, and George M. Whitesides. 2014. "Charging of Multiple Interacting Particles by Contact Electrification." Journal of the American Chemical Society 136 (38) (September 24): 13348-13354. doi:10.1021/ja506830p.

\section{Published Version}

doi:10.1021/ja506830p

\section{Permanent link}

http://nrs.harvard.edu/urn-3:HUL.InstRepos:16732680

\section{Terms of Use}

This article was downloaded from Harvard University's DASH repository, and is made available under the terms and conditions applicable to Open Access Policy Articles, as set forth at http:// nrs.harvard.edu/urn-3:HUL.InstRepos:dash.current.terms-of-use\#OAP

\section{Share Your Story}

The Harvard community has made this article openly available.

Please share how this access benefits you. Submit a story.

Accessibility 


\section{Charging of Multiple Interacting Particles by Contact Electrification}

Siowling Soh, ${ }^{a}$ Helena Liu, ${ }^{a}$ Rebecca Cademartiri, ${ }^{a}$ Hyo Jae Yoon, ${ }^{a}$ George M. Whitesides* ${ }^{a b c}$

${ }^{a}$ Department of Chemistry and Chemical Biology, Harvard University, 12 Oxford Street, Cambridge, MA 02138, USA

${ }^{b}$ Wyss Institute for Biologically Inspired Engineering, Harvard University, 60 Oxford Street, Cambridge, MA 02138, USA

${ }^{c}$ Kavli Institute for Bionano Science and Technology, School of Engineering and Applied

Sciences, Harvard University, 29 Oxford Street, Cambridge, MA 02138, USA

* Corresponding author: gwhitesides@gmwgroup.harvard.edu 


\begin{abstract}
Many processes involve the movement of a disordered collection of small particles (e.g., powders, grain, dust, and granular foods). These particles move chaotically, interact randomly among themselves, and gain electrical charge by contact electrification. Understanding the mechanisms of contact electrification of multiple interacting particles has been challenging, in part due to the complex movement and interactions of the particles. To examine the processes contributing to contact electrification at the level of single particles, a system was constructed in which an array of millimeter-sized polymeric beads of different materials were agitated on a dish. The dish was filled almost completely with beads, such that beads did not exchange positions. At the same time, during agitation, there was sufficient space for collisions with neighboring beads. The charge of the beads was measured individually after agitation. Results of systematic variations in the organization and composition of the interacting beads showed that three mechanisms determined the steady-state charge of the beads: (i) contact electrification (charging of beads of different materials), (ii) contact de-electrification (discharging of beads of the same charge polarity to the atmosphere), and (iii) a long-range influence across beads not in contact with one another (occurring, plausibly, by diffusion of charge from a bead with a higher charge to a bead with a lower charge of the same polarity).
\end{abstract}




\section{Introduction}

When a collection of particles with different composition move and collide randomly among themselves, they tend to acquire an electrical charge; the processes that cause charging are called, collectively, “contact electrification”. ${ }^{1-3}$ Contact electrification of multiple interacting particles is a phenomenon that has many important consequences. It can have a powerful influence over natural phenomena (e.g., generation of lightning, the behavior of sand storms, and initiation of dust explosions). ${ }^{4}$ In industry, contact electrification can be either problematic or beneficial; for example, it leads to aggregation of particles on the walls of vessels and reactors (and thus hinders efficient heat transfer), and non-uniform blending of pharmaceutical products when the particles agglomerate or segregate; it also underlies processes such as electrophotography, electrostatic coating with powders, and electrostatic precipitation and spray painting. ${ }^{1,4}$

Research on mechanisms of contact electrification of multiple particles is challenging for a number of reasons — these mechanisms are intrinsically dynamic, and the history of their contributions to accumulated charge depend on parameters that are not necessarily easily controlled (e.g., pressures at contacting asperities). In particular, contact electrification involves multiple mechanisms; there is no experimental system that allows the charge of the particles (or charging regions) to be measured individually at all relevant dimensional scales, or the type of interaction between particles to be controlled systematically. This paper describes a system that consists of an array of millimeter-sized beads, composed of different polymers, moving on a uniform surface in a dish; this system was designed as a model with which to investigate the mechanisms determining the steady-state charge of multiple particles during contact electrification. 
Previous studies have suggested that contact electrification of insulating materials is a complex process that may involve several different species (e.g., ions and radicals), and processes (e.g., ion transfer, electron transfer, and material transfer) operating simultaneously. ${ }^{3-6}$ When two solid surfaces are brought into contact and then separated, there is a tendency for one surface to gain a net positive charge, while the other surface gains a net negative charge (Figure 1). This separation of charge suggests the transfer of charged species (which may be a single type of mobile ion, a mixture of ions, a mixture of ions and electrons, or microscopic fragments of charged matter) from one surface to another. For contacts between two metallic surfaces, the species that carries charge is assumed to be an electron. For two insulating surfaces, the charged species transferred during contacts are predominantly ions (at least for low levels of charge transferred). ${ }^{3,7}$ For example, ions are transferred during contact electrification when one solid surface is coated with a layer composed of covalently bound, immobile, ionic groups and mobile counterions. ${ }^{8,9}$ Here, the mobile counterions are clearly the species transferred from one surface to another after contact. It is, therefore, probable that this mechanism applies generally to contact electrification of any type of insulating materials. When the process that transfers charge also involves (or correlates with) a transfer of a large amount of material (that is, quantities at the microscopic scale) with attached ionic groups, it is known as “material transfer".,10

A number of studies have shown that when a surface is sufficiently charged during contact electrification, it can also discharge,,$^{2,4,11,12}$ and it is the relative rates of the charging and discharging that establish the steady-state charge. Discharge is strongly influenced by the dielectric breakdown strength of the atmosphere (e.g., 3 MV/m for air $^{13}$ ). When a particle charges highly due to contact electrification, the electric field around the particle may exceed the dielectric breakdown strength of air. In this circumstance, neutral gas molecules surrounding the 
Figure 1. Contact electrification and contact de-electrification. Contact electrification is the phenomenon in which a solid surface gains a net positive charge while another solid surface gains a net negative charge after the two surfaces are brought into contact and are then separated. Contact de-electrification is the phenomenon where two initially charged solid surfaces — and with the same charge polarity — discharge when the surfaces are brought into contact. Charge is transferred from the solid surfaces to the gaseous atmosphere (presumably to the molecules of gas in the air).
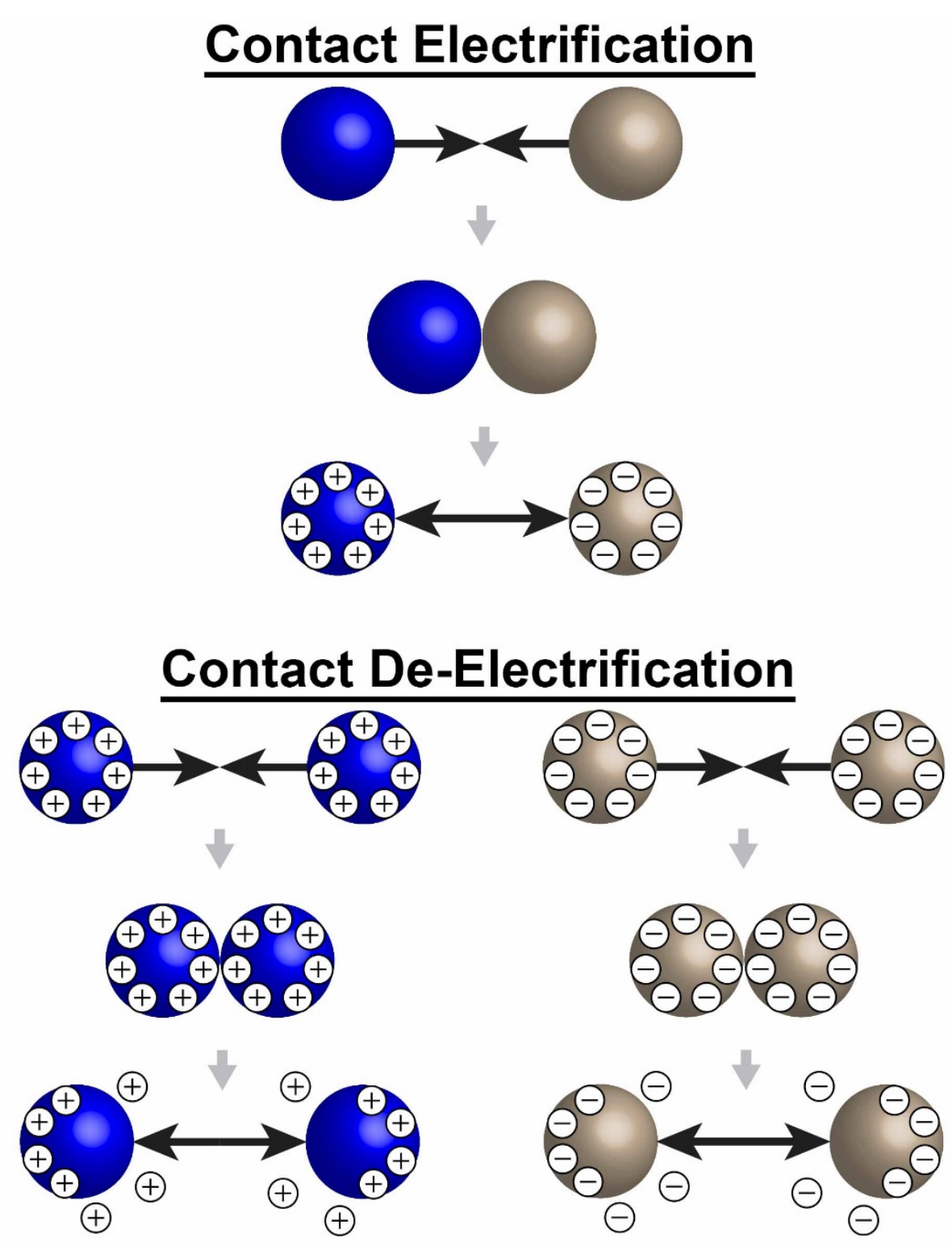
particle ionize. This process can produce a local corona, and result in loss of charge to the atmosphere.

We have now examined this process, and found that when two charged surfaces of the same polarity were brought into contact, they discharged (Figure 1) ${ }^{14}$ This result was initially surprising since it involved loss of charges from both the contacting surfaces. This process which we call contact de-electrification ${ }^{14}$ — occurs when two surfaces of the same polarity are brought into contact, and the local electric field at the point of contact (a combination of the electric fields from both the surfaces) is higher than the dielectric breakdown strength of the surrounding atmosphere. In this case, the surrounding gas molecules ionize and partly discharge the surfaces by transferring charge to molecules in the air.

Contact electrification is typically studied by examining the physical contact of two solid surfaces; this contact can be performed by manually contacting and separating two solid objects, ${ }^{15}$ rolling a bead on a solid surface, ${ }^{9,16,17}$ or colliding a particle with a solid surface. ${ }^{18}$ Methods used for studying contact electrification of many particles include allowing them to slide down a solid surface by gravity, ${ }^{19}$ vibrating them on a solid surface, ${ }^{20}$ forcing them through a pipe via the flow of a carrier gas, ${ }^{21,22}$ or agitating them in a fluidized bed. ${ }^{23,24}$ These methods, however, typically involve a large number of particles. These particles tend to move chaotically and collide with each other in an unpredictable manner; thus, it is difficult to study the mechanisms related to contact electrification at the level of a single particle. In order to understand the phenomenon at the single-particle level, we wished to have an experimental system that allows (i) physical interaction among multiple particles, (ii) measurement of charge of a particle within the interacting particles, and (iii) control of the specific types of physical contact made by each particle. 
In this study, we used macroscopic (millimeter-sized) polymeric beads to investigate the mechanisms influencing contact electrification of multiple particles. The beads were allowed to move and interact with each other by placing them on a dish, which was agitated by a motor. The use of macroscopic beads allowed us to distinguish, handle, and measure the charge on each bead easily.

We designed the system such that it gave us the capability to control the types of interactions systematically that each beads made with other beads. Our results show that three mechanisms significantly influence the steady-state charge of the beads: contact electrification, contact de-electrification, and a long-range influence across layers of beads (e.g., probably diffusion of ions across multiple beads facilitated by rotation of the beads; but since we have no direct evidence for a specific mechanism, we use the more general term "long-range influence").

\section{Experimental Section}

We studied the interaction among multiple interacting millimeter-sized (i.e., 1/4 inch diameter) polymeric beads (McMaster-Carr) rather than smaller particles, so that each individual particle could be easily distinguished, tracked, and manipulated. We used polyamide 6/6 (Nylon; charges positively) and polytetrafluoroethylene (Teflon; charges negatively) beads as they could be charged highly according to the triboelectric series (an empirically ordered list of materials that ranks the tendency of a material to charge either positively or negatively). ${ }^{25}$ In order to differentiate the colorless beads, we dyed the Nylon beads blue (Disperse Blue dye from SigmaAldrich); we showed in the Supporting Information, SI, (Figure S1) that dyed and undyed Nylon beads behaved similarly. All beads were rinsed with deionized water and ethanol several times 
before use in order to remove any foreign particles on their surface. The dish used was either aluminum (square, inner length $8.9 \mathrm{~cm}$ ), Nylon (circular, diameter $9 \mathrm{~cm}$ ), or Teflon (circular, diameter $9 \mathrm{~cm}$ ). We used polybutylene terephthalate tweezers (McMaster-Carr) for all manipulations of the beads; we demonstrated previously ${ }^{14}$ that picking up charged beads with these tweezers did not affect their charge. In order to eliminate the influence of changes in humidity and levels of dust or organic contaminants, we conducted all the experiments in a glove bag, under a nitrogen atmosphere, and at a humidity of 1-2\% (at room temperature).

\section{Results and Discussion}

The experimental methods and materials used in this work have been described in previous papers for investigating the electrostatic self-assembly of beads. ${ }^{26,27}$ Our strategy in this work differed from previous studies in which we mixed two types of spheres, and correlated tribocharging with the emergence of crystalline order. Here, we arranged spheres in crystalline patterns that allowed them enough mobility to charge when agitated, but not enough to change their positions in the lattice. Figure 2 shows the packing of the beads: they are arranged in a hexagonal lattice with 13 rows and 16 columns in the dish.

Before the experiment, the beads were discharged by spraying neutralizing ions onto them from an antistatic "Zerostat” gun (VWR) (in a separate experiment, we measured the charges on the beads after this process, and found it to be negligible; this result confirmed that the beads were indeed discharged by the ions from the antistatic gun.) This neutralizing step was conducted for all the experiments involved in this study. In the first experiment, we filled the dish with all Teflon beads, except for one Nylon bead in the center of the dish. The beads and the 
Figure 2. Experiment for investigating the mechanisms influencing contact electrification of multiple interacting polymeric beads. a) In this scheme, a dish filled with beads is agitated linearly by a motor. The dish is filled with an excess of one type of beads (e.g. Teflon) while a bead in the center of the dish is of a different material (e.g. Nylon). After agitation, the charge on the bead in the center of the dish is measured using a Faraday Cup connected to an electrometer. b) Actual experimental images showing the cases in which there is an excess number of Teflon beads (left image) and an excess number of Nylon beads (right image, Nylon beads are dyed blue for clarity) in dishes made of aluminum. 

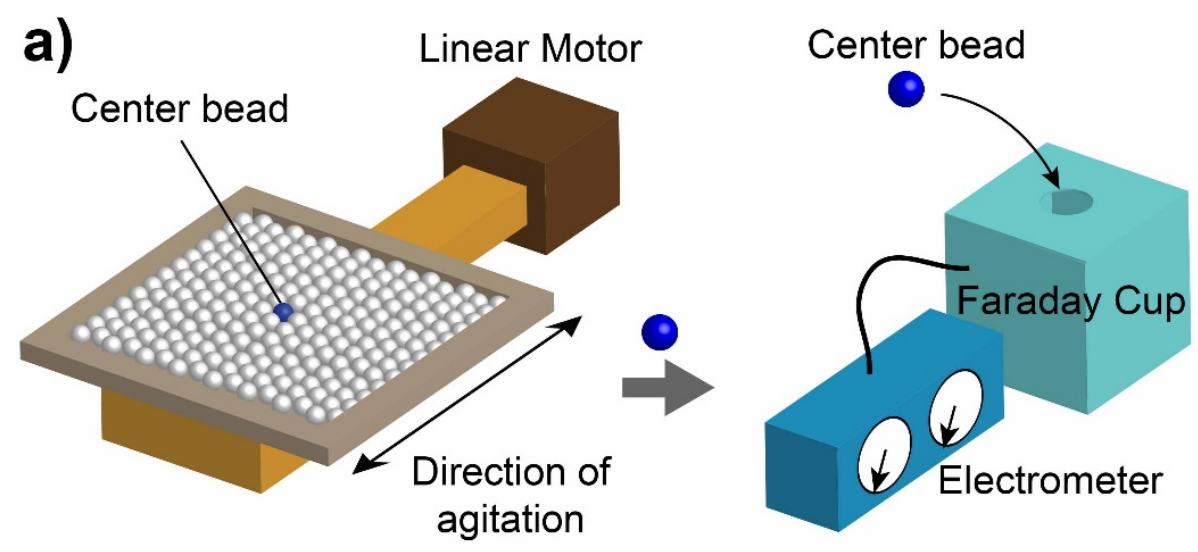

b)

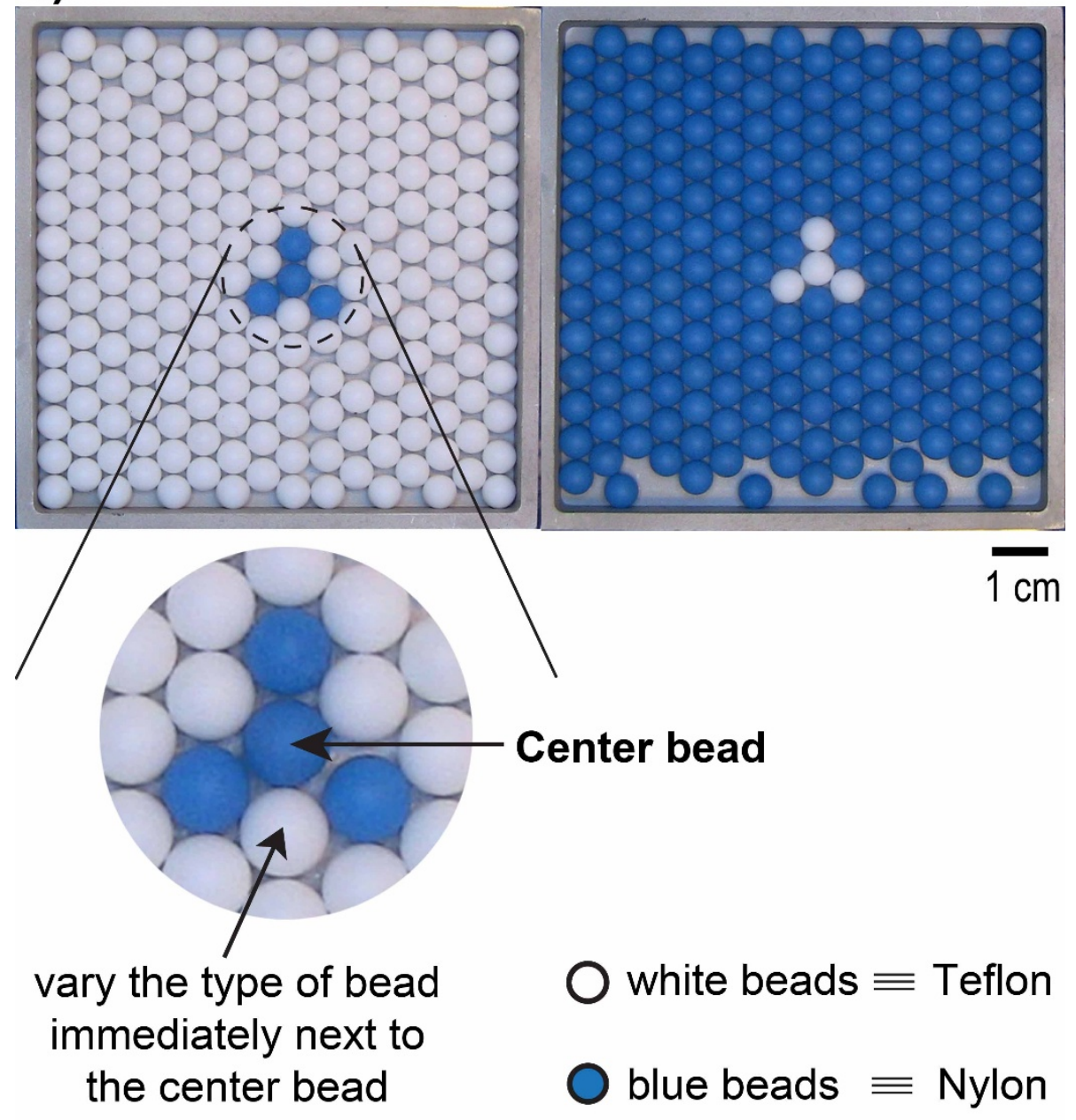


dish were then agitated linearly (direction as shown in Figure 2a) using a motor for 3 mins. The motor operated at a maximum displacement of $9 \mathrm{~cm}$, and a frequency of $6 \mathrm{~Hz}$. The beads rolled and collided with each other in the course of the agitation; these interactions among the beads allowed us to study the various mechanisms of contact electrification. Since the dish was almost completely filled with beads, each bead contacted only the same six neighboring beads during agitation. After agitation, the bead in the center of the dish was transferred into a Faraday Cup. The Faraday Cup (made in-house, cubic with length of $10 \mathrm{~cm}$ ), connected to an electrometer (Keithley, model 6514), measured the charge of the bead placed within the Cup (Figure 2a).

In order to examine the influence of the beads surrounding the center Nylon bead on the charge of the center bead, we sequentially changed the six neighboring Teflon beads to Nylon beads one-by-one; for each interchange, we repeated the procedure as described above. The experimental image on the left in Figure 2b shows the case where three Teflon beads were replaced with Nylon beads. We also repeated the entire experiment by interchanging Teflon and Nylon beads, and Nylon and Teflon beads (e.g., image on the right in Figure 2b). Finally, we again repeated the whole set of experiments described above twice, using dishes made of Teflon or Nylon (instead of aluminum).

\section{Charge Decreases with Number of Beads of the Same Type}

Figure 3 shows the results of the experiments. The seven beads shown on the $x$-axis represent the type of center bead and its six neighbors used (blue represents Nylon, white represents Teflon). For all the experiments conducted, the trend is universal: for an increasing number of neighbors of the same material as the center bead, the steady-state charge on the center bead decreases. 
Figure 3. Influence of the six neighboring beads on the charge of the center bead. Plots of the charge density of the center bead when it is agitated against neighboring beads of the same or different material as indicated by the $x$-axis (blue circles represent Nylon beads and white circles represent Teflon beads). Other than the seven beads indicated on the $x$-axes, the rest of the beads on the dish are always of a different material than the center bead. Data denoted by solid black circles indicate the case in which the center bead is Nylon $(n=7-12)$; open circles indicate the case in which the center bead is Teflon $(n=7-12)$. Beads are agitated on a) an aluminum dish, b) a dish of a different material as the center bead, and c) a dish of the same material as the center bead. The plots show that the charge on the center bead decreases when there are more beads of the same material surrounding the center bead. These trends are the same for beads agitated on dishes of different materials. 

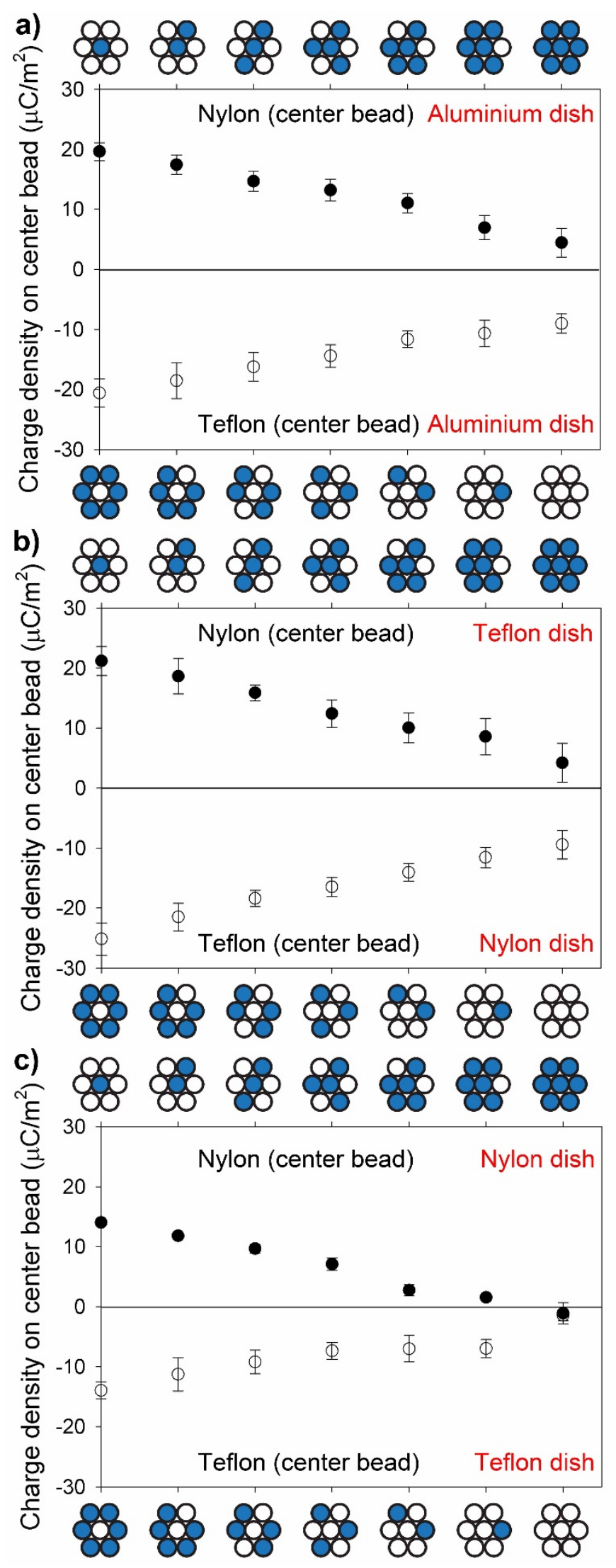
The type of dish used (i.e., aluminum, Nylon, or Teflon) seems to affect only the absolute charge on the center bead but not the decreasing trend shown in Figure 3. According to the triboelectric series, a Nylon (or Teflon) bead is expected to charge most highly when in contact with a Teflon (or Nylon) dish, followed by an aluminum dish, and then a Nylon (or Teflon) dish. The results we obtained are in agreement with the expectations from the triboelectric series: ${ }^{25}$ the charge of the center bead shown in Figure 3b (Nylon bead with Teflon dish or Teflon bead with Nylon dish) is either slightly more charged, or similar in charge, when compared to the charge of the center bead shown in Figure 3a (Nylon bead with aluminum dish or Teflon bead with aluminum dish). Figure 3c (Nylon bead with Nylon dish or Teflon bead with Teflon dish) shows the lowest charge among the three plots. The decreasing trend, however, for all three plots is the same - this result shows that the material of the dish does not affect the trend observed.

Several more observations help to clarify the processes occurring in these experiments. i) In a separate experiment, we established that the beads rotated in different directions during agitation by marking a few areas of the surface of the beads with different colors, and observing the rotations. ii) The charge on the center bead reached steady state after 3 mins of agitation. The results were similar when we agitated the dish for either 3 mins or 10 mins (see SI, Figure S2). iii) The charge on the center bead depended on the number of beads of the same (or different) materials, but not on the disposition of these beads around the center bead. Figure 4 shows three other configurations possible for the case when the center Teflon bead is surrounded by either four, three, or two Nylon beads. The charges on the center bead are similar for the three different configurations for the same number of Nylon beads, but are significantly different across different numbers (i.e., four, three, or two) of Nylon beads. iv) We showed previously ${ }^{14}$ that a charged bead, when left undisturbed, retained its charge for extended periods of time (i.e., 
hours); this observation shows that the charge on the bead does not dissipate readily by itself (e.g., into the atmosphere).

The results from these observations are difficult to reconcile with a process in which the only operative mechanism is contact electrification. Take, for example, the case where a Teflon bead is surrounded by six Nylon beads, and another case where a Teflon bead is surrounded by four Nylon beads. In each case, the Teflon bead rolls, rotates, and charges against other Nylon beads until it attains a steady-state charge. If contact electrification were the only important mechanism, we would expect the Teflon beads to have similar limiting charge at steady-state for both cases. In fact, our experiment shows that the charge of the Teflon bead surrounded by six Nylon beads is higher than when it is surrounded by four Nylon beads. This observation suggests that there is a loss of charge when the center bead is surrounded by beads of the same material.

We believe that the trend to decrease the charge on the center bead with an increase in the number of neighbors of the same composition is the result of the interplay of two effects: contact electrification and contact de-electrification. When the center bead is surrounded by more neighbors of a different material, it charges more highly due to contact electrification. When the center bead is surrounded by more neighbors of the same material, it discharges more due to contact de-electrification between like-charged beads.

\section{Gain and Loss of Charge}

Both the gain and the loss of charge can be demonstrated clearly by measuring the different charges on a same center bead, surrounded by different types of neighbors. Specifically, we first filled a Nylon dish with all Nylon beads except for seven Teflon beads; that is, only the bead at 
Figure 4. Exact configuration of the beads is less important than the number of each type of bead surrounding the center bead. The graph plots the charge density of the center bead for, from left to right, four, three, and two Nylon beads surrounding the center Teflon bead. Each of these numbers of Nylon beads contains three distinct possibilities for positioning the beads around the single Teflon bead. The dish used was Nylon, and the rest of the beads in the dish were Nylon. Results show that the charge of the center bead is similar for different configurations of the Nylon beads, but is different for different numbers of Nylon beads surrounding the center bead. $(n=7-12)$

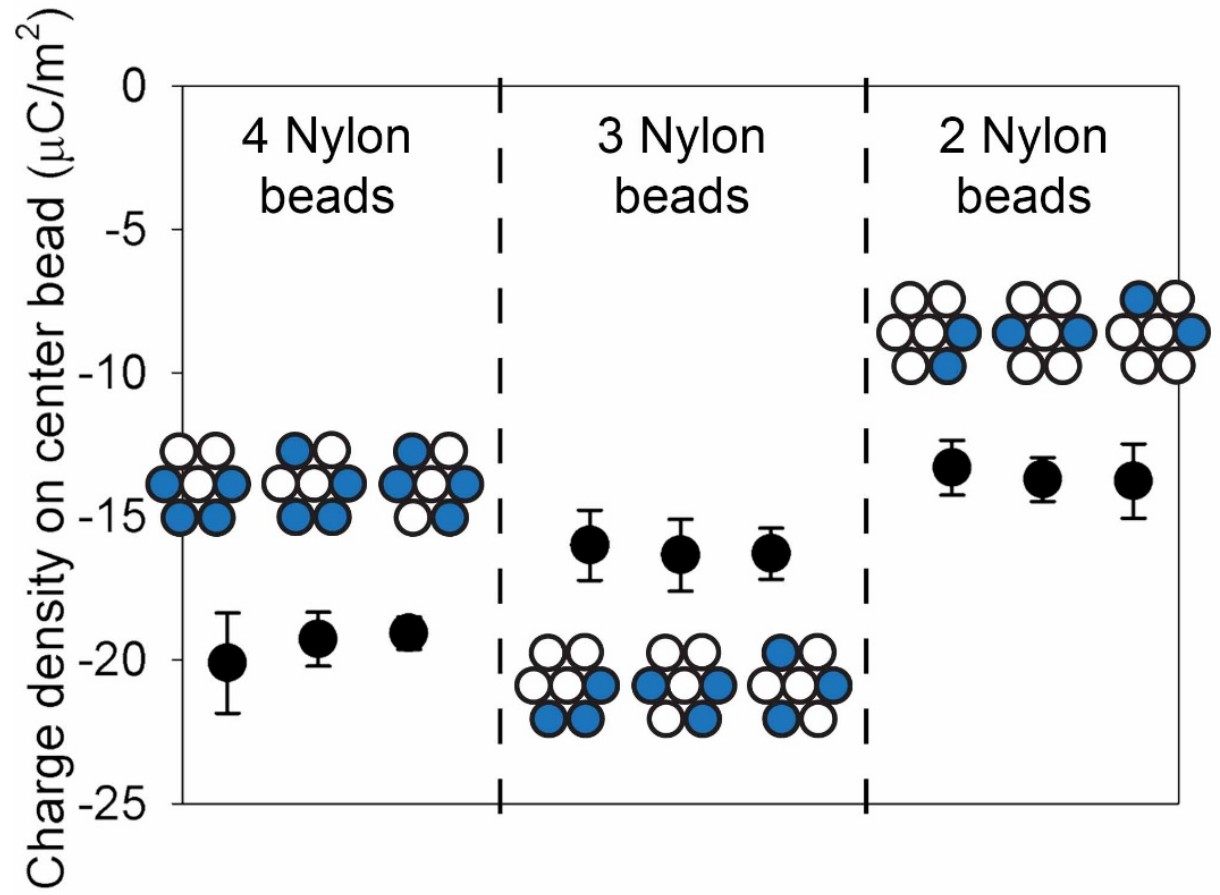


the center of the dish and its six neighbors were Teflon. After agitating the dish for 3 mins and measuring the charge on the center bead, we found that it charged only slightly negatively, as observed in previous experiments. We then changed the six neighboring Teflon beads to Nylon, while leaving the same center bead in the dish. After agitating the dish and measuring the charge, we found that the charge on the center bead increased (Figure 5). This result is an (obvious) demonstration of contact electrification.

We also performed the experiment in reverse: the center Teflon bead was initially surrounded by six Nylon beads rather than six Teflon beads. (We used the same Nylon dish, and Nylon beads for filling the rest of the dish.) After agitating the dish, a high charge was found on the center bead. After the six neighboring beads were changed to Teflon and the dish was agitated again, the charge on the center bead decreased (Figure 5) — a clear demonstration that charge (originally on the center bead after agitating the dish the first time) was lost from the center bead.

This loss of charge cannot be explained by a transfer of charge from the center bead to the dish because the center Teflon bead was in contact with a dish made of Nylon. Since Teflon charges highly with Nylon, the Teflon bead should gain, and not lose charge through contact with the Nylon dish. Also, the Teflon bead interacted with the same dish in the same way for both experiments; the only change is the number of Nylon and Teflon beads surrounding the center bead. Hence, we propose that the loss of charge is due to contact de-electrification involving transfer of charge from contacting like-charged beads to the atmosphere. When a Teflon bead is surrounded by six Nylon beads, only contact electrification takes place among the beads, thus charging the center bead highly. When a Teflon bead is surrounded by one or more 
Figure 5. Demonstration of both charging and discharging when beads of the same and different materials interact with one another. A Teflon bead was used as the center bead; the dish used was Nylon. When this center Teflon bead was originally surrounded by six neighboring Teflon beads (the rest of the beads on the dish were Nylon), it developed only a slight negative charge when the dish was agitated. When the six neighboring beads were replaced with Nylon beads and the dish was agitated again, the charge on the same center bead increased. The opposite is true. In a separate experiment, the center Teflon bead was initially charged highly due to its interaction with six neighboring Nylon beads (the rest of the beads on the dish were also Nylon). After changing the six Nylon beads to Teflon beads and agitating the dish again, the charge on the same center bead decreased. This dependence of the extent of charging illustrates the sensitivity of steady-state charge on the balance of contact electrification and contact de-electrification. $(n=$ 7)

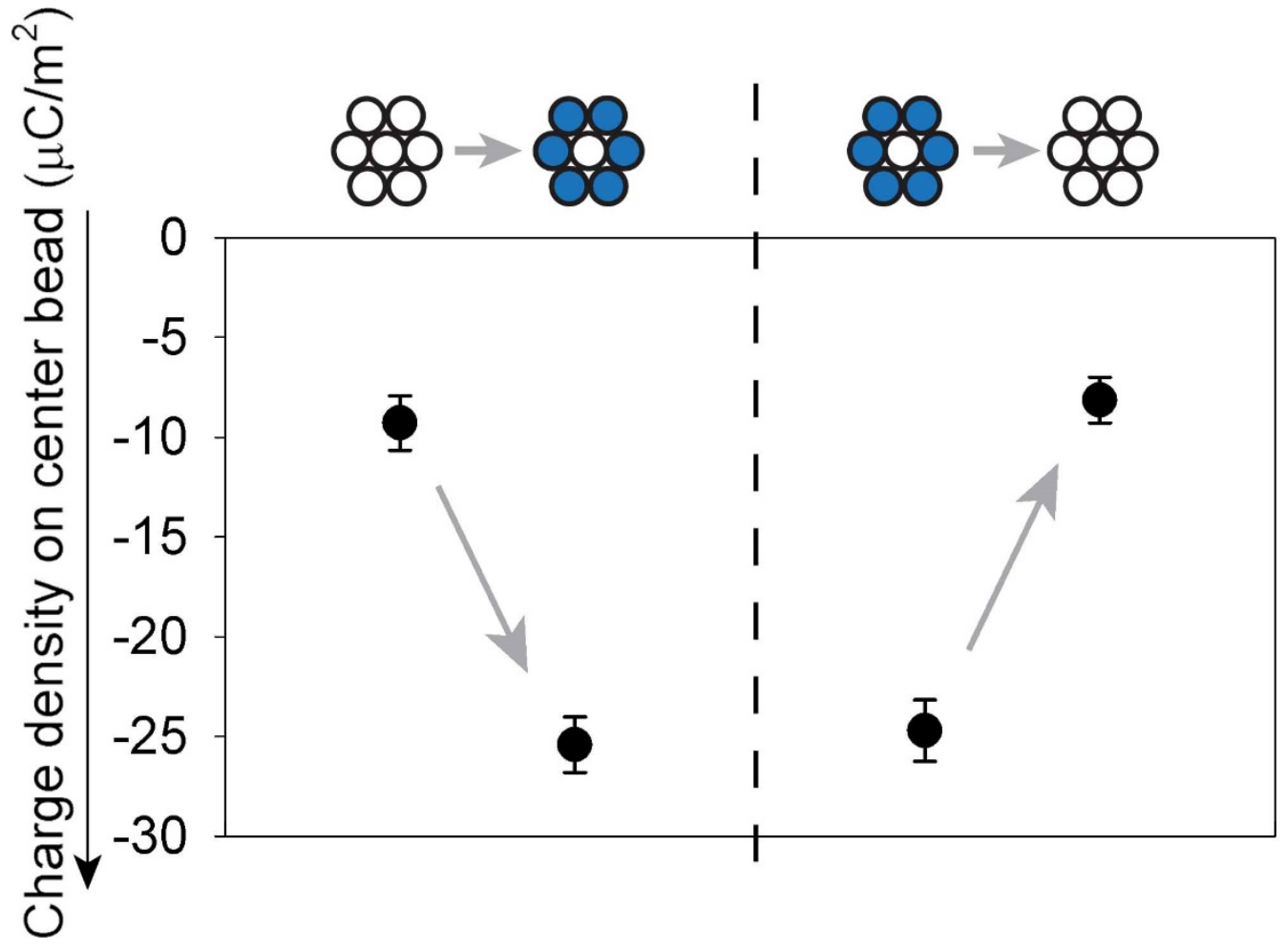


Teflon beads, contact de-electrification among the Teflon beads causes the center bead to discharge.

One other possibility, however, remains: is it possible that charge on the center bead decreases as a result of transfer of charge from the center bead to its neighbors of the same polymer?

\section{Long-range Influence of Beads on the Outer Layers on the Charge of the Center Bead}

In order to investigate the transfer of charge between beads of the same material, we performed two types of experiments as follows. In the first, we filled an aluminum dish with only Teflon beads (left scheme of Figure 6a), and measured the charge on a selected bead (the "center bead") after agitating the dish. In the second, we changed all the beads in the dish to Nylon except that center bead and its six neighbors (right scheme of Figure 6a). Unexpectedly (for a model in which only nearest neighbors interact), the charges on the center beads are different for the two types of experiments (Figure 6a); that is, the charges are statistically different at a 99\% confidence level (or $p<0.01$; results were calculated using Matlab 2010b). Although the "center" Teflon beads were in contact with six Teflon beads in both experiments — we changed only the beads that were not in contact with the center bead — the charge on this bead was slightly different. It is thus surprising that the charges of the center beads were different even though the center beads were in contact with the same type of beads and dish in both experiments. We repeated the experiment with a Nylon dish (instead of an aluminum dish), and found similar results (Figure 6a). For a closer examination of the phenomenon, we varied the beads next to, but at an outer layer of, the six neighboring beads as shown in the schemes in 
Figure 6. Long-range influence of the beads not in contact with the center bead on the charge of the center bead. Teflon beads were used as the center beads, and the six neighboring beads next to the center beads were also always Teflon. The material used for the rest of the beads on the dish depends on the specific experiment, and are indicated by the color of the dotted circles (blue represents Nylon, while white represents Teflon). a) When the rest of the beads were Nylon, the center bead charged significantly higher than when the rest of the beads were Teflon (* represents $p<0.01$ ). The experiments were performed for dishes made of aluminum and Nylon. b) A systematic variation of the beads in the outer layer surrounding the six neighboring beads was conducted. In general, there was an increase in the charge of the center bead when the six neighboring beads were surrounded by more Nylon beads. The dish used in this case was aluminum. (* represents $p<0.01$, and + represents $p<0.05)(n=7-15)$ 
a

No charge transfer

Charge transfer to
center bead
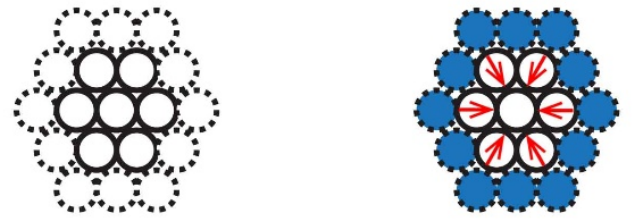

Rest of the beads on the dish: Teflon Nylon
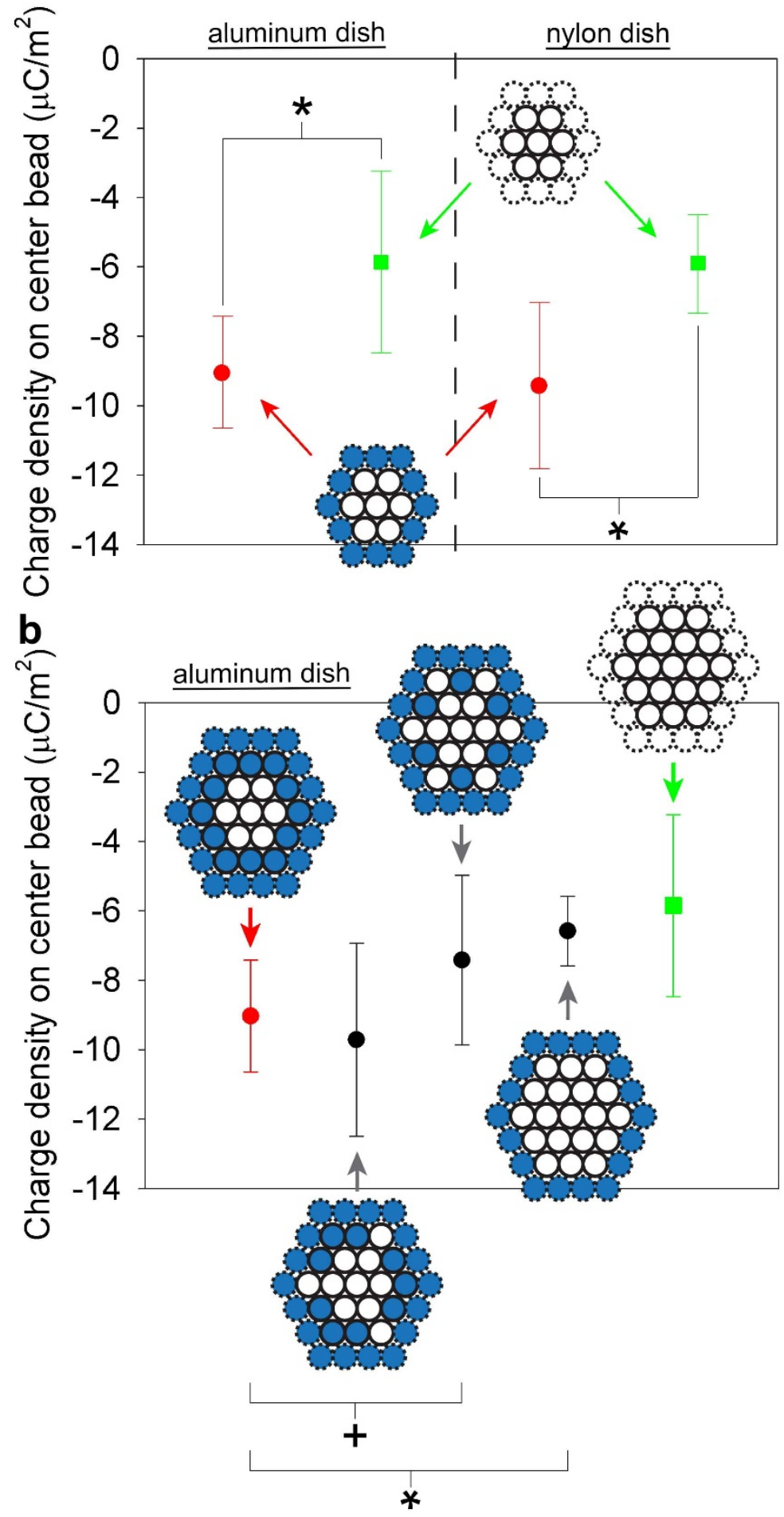
Figure 6b. In general, the charge of the center Teflon bead increases when there are more Nylon beads in the outer (second) layer.

There are at least two possible explanations to these observations: i) Charge Diffusion. In this explanation, there would be a diffusion of charge from the neighboring beads to the center bead on contact (see scheme on the top right of Figure 6a). When there are more Nylon beads in the outer layer, each of the six Teflon neighbors contacts more Nylon beads; these interactions lead to a higher charge on the six Teflon neighbors. It is then possible for charge to transfer from the higher charged neighbors to the lower charged center bead on physical contact. Importantly, this mechanism suggests that charge transfers only from a surface of higher charge to a surface of lower charge, and not vice versa. This type of process would resolve our question raised in the previous Section: the loss of charge from the center bead cannot be due to a transfer of charge from the center to its neighboring beads, which charged either similarly or higher than the center bead for all the configurations considered. If there was a transfer of charge from a highercharged surface to a lower-charged surface, it was, however, not always significant. When the beads were arranged in a different configuration (e.g., in a line of Nylon beads as shown in the SI, Figure S3), the difference was not sufficiently statistically significant to conclude that there was a transfer of charge. ii) Electrostatic Destabilization. In this rationalization, the overall electrostatic field gradient around the contact point in the cluster (relative to some more distant point in the gas phase) would depend on the size of the aggregate of like-charged beads. Larger aggregates (in this mechanism) would tend to discharge more rapidly to the surrounding gas than smaller aggregates. 


\section{Conclusion}

We constructed an experimental system for investigating the mechanisms involved in contact electrification of multiple interacting beads. In particular, we are able to examine the charge of an individual bead, and vary the type of interaction between beads of different or same materials systematically. In a cluster of beads, the charge at steady state on a bead decreases as the number of nearest neighbors of the same material increases. (Beads in the second neighboring layer have a much smaller, but still significant, charge.) We propose that this trend is due to contact deelectrification: when the electric field at the region of contact between beads of the same charge polarity exceeds the dielectric breakdown strength of air, charge transfers from the solid surfaces to gas molecules in the atmosphere. We do not know the mechanism of this charge transfer: formation of a plasma, charge transfer along ionized tracks formed by radioactive decay (most plausibly of $\mathrm{K}^{+}$) or cosmic rays, or a number of other idiosyncratic processes are all possible. Regardless of the detailed mechanism, when a bead is in contact with more beads of the same materials (and the same charge polarity), it discharges more; this discharge leads to a lower steady-state charge. This result illustrates the importance of contact de-electrification among multiple interacting particles - a bead can discharge significantly, as long as it is in contact with a single bead of the same charge polarity.

The decreasing trends (shown in all the plots in Figure 3) are approximately linear. One explanation for the linear trend is that the mechanisms related to contact electrification take place only at the surface of the beads: the surface area of the neighboring beads of a different (or same) material varies linearly across the $x$-axes of the plots in Figure 3. If the amount of contact electrification and de-electrification varies proportionally with the surface area of the types of beads involved, we can expect the trend to be linear. 
Besides charging and discharging, we also showed that there is a long range influence of the beads on their charges: that is, the charge on the center bead changed, when the material of the beads not in direct contact with the center bead changed.

To summarize, we found that three types of phenomena influence the steady-state charge of the beads: contact electrification (charging between surfaces of different materials), contact de-electrification (discharging between surfaces of the same charge polarity), and a long-range effect (e.g., the transfer of charge through contact from a bead with a higher charge to a bead with a lower charge).

\section{Acknowledgement}

This work was funded by the Department of Energy, Division of Materials Sciences and Engineering under award \#ER45852. H.L. was funded by the Harvard REU program under NSF award \#DMR-0820484.

\section{Associated Content}

Supporting Information. Comparing the charge of dyed and undyed Nylon beads after contact electrification, establishing that three minutes is sufficient for a bead to reach steady-state charge, and showing the lack of significant diffusion of charge between beads when they are arranged in a particular configuration in a dish. This material is available free of charge via the Internet at http://pubs.acs.org. 


\section{References:}

(1) Matsusaka, S.; Maruyama, H.; Matsuyama, T.; Ghadiri, M. Chem. Eng. Sci. 2010, 65, 5781.

(2) Harper, W. R. Contact and Frictional Electrification; Oxford University Press: London, 1967.

(3) McCarty, L. S.; Whitesides, G. M. Angew. Chem., Int. Ed. 2008, 47, 2188.

(4) Lacks, D. J.; Sankaran, R. M. J. Phys. D: Appl. Phys. 2011, 44, 453001.

(5) Baytekin, H. T.; Baytekin, B.; Hermans, T. M.; Kowalczyk, B.; Grzybowski, B. A. Science 2013, 341, 1368.

(6) Baytekin, H. T.; Baytekin, B.; Incorvati, J. T.; Grzybowski, B. A. Angew. Chem., Int. Ed. 2012, 51, 4843.

(7) Diaz, A. F.; Fenzel-Alexander, D. Langmuir 1993, 9, 1009.

(8) McCarty, L. S.; Winkleman, A.; Whitesides, G. M. Angew. Chem., Int. Ed. 2007, 46, 206.

(9) McCarty, L. S.; Winkleman, A.; Whitesides, G. M. J. Am. Chem. Soc. 2007, 129, 4075.

(10) Baytekin, H. T.; Patashinski, A. Z.; Branicki, M.; Baytekin, B.; Soh, S.; Grzybowski, B.

A. Science 2011, 333, 308.

(11) Thomas, S. W.; Vella, S. L.; Kaufman, G. K.; Whitesides, G. M. Angew. Chem., Int. Ed. 2008, 47, 6654.

(12) Vella, S. J.; Chen, X.; Thomas, S. W.; Zhao, X. H.; Suo, Z. G.; Whitesides, G. M. J. Phys. Chem. C 2010, 114, 20885.

(13) CRC Handbook of Chemistry and Physics; 90 ed.; Lide, D. R., Ed.; CRC Press: Boca Raton, 2010.

(14) Soh, S.; Kwok, S. W.; Liu, H.; Whitesides, G. M. J. Am. Chem. Soc. 2012, 134, 20151. 
(15) Lowell, J.; Roseinnes, A. C. Adv. Phys. 1980, 29, 947.

(16) Wiles, J. A.; Grzybowski, B. A.; Winkleman, A.; Whitesides, G. M. Anal. Chem. 2003, 75, 4859.

(17) Rowley, G. Int. J. Pharm. 2001, 227, 47.

(18) Watanabe, H.; Ghadiri, M.; Matsuyama, T.; Ding, Y. L.; Pitt, K. G. Rev. Sci. Instrum. 2007, 78, 024706.

(19) Oguchi, T.; Tamatani, M. Wear 1993, 168, 91.

(20) Higashiyama, Y.; Ujiie, Y.; Asano, K. J. Electrost. 1997, 42, 63.

(21) Matsusaka, S.; Fukuda, H.; Sakura, Y.; Masuda, H.; Ghadiri, M. Chem. Eng. Sci. 2008, 63, 1353.

(22) Gajewski, A. J. Electrost. 1989, 23, 55.

(23) Iuga, A.; Calin, L.; Neamtu, V.; Mihalcioiu, A.; Dascalescu, L. J. Electrost. 2005, 63, 937.

(24) Zhao, H.; Castle, G. S. P.; Inculet, I. I. J. Electrost. 2002, 55, 261.

(25) Diaz, A. F.; Felix-Navarro, R. M. J. Electrost. 2004, 62, 277.

(26) Grzybowski, B. A.; Winkleman, A.; Wiles, J. A.; Brumer, Y.; Whitesides, G. M. Nat. Mater. 2003, 2, 241.

(27) Cademartiri, R.; Stan, C. A.; Tran, V. M.; Wu, E.; Friar, L.; Vulis, D.; Clark, L. W.; Tricard, S.; Whitesides, G. M. Soft Matter 2012, 8, 9771. 
Table of Content Only

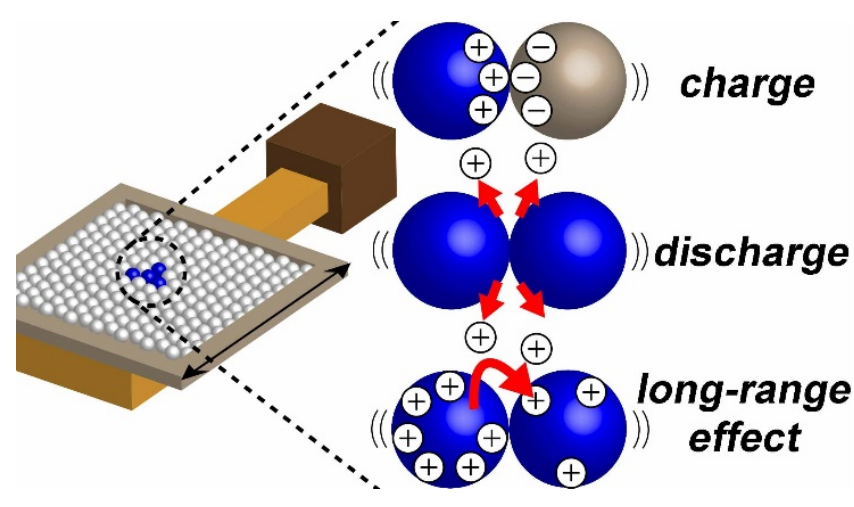

